\title{
THE ACCURACY OF SPIROGRAPHIC RECORDING AT HIGH RESPIRATORY RATES
}

\author{
BY \\ L. BERNSTEIN AND D. MENDEL \\ From the Physiology Department, London Hospital Medical College
}

(RECEIVED FOR PUBLICATION MARCH 27, 1951)

Spirometers were designed and used originally for the measurement of metabolic rate or such respiratory constants as vital capacity. Such measurements are made at low rates of respiration (up to about 40 breaths per minute) or with single inspiratory or expiratory efforts. There has been no reason to suspect the accuracy of records made under these conditions.

Since Hermannsen (1933) first described the maximum breathing capacity test (M.B.C.) many investigators have used the spirometer to record tidal air at considerably higher rates of breathing. During an investigation into the M.B.C. of a series of subjects D'Silva and Mendel (1950) noted that at respiratory rates higher than 55 breaths per minute the oscillatory movements of the water in their spirometer were of such violence that very often water was aspirated into the tubes connecting the subject to the instrument, and that aspiration invariably occurred at these high respiratory rates if the tidal air was large. The large movements of the water column which they noted must have been either the cause, or the result, of considerable pressure changes within the spirometer bell, and it seemed that these pressure changes might affect the excursions of the bell and so invalidate the M.B.C. estimations. It was decided therefore to investigate the response of the spirometer at various respiratory rates in order to determine: (a) the magnitude of the errors produced, if any, and $(b)$ whether it would be possible to construct a calibration curve for the instrument so that the errors could be corrected.

\section{DesCription OF THE SPIROMETER}

This was the same instrument as that used by D'Silva and Mendel in their experiments. It is of the Knipping type (manufactured by C. F. Palmer), and the $\mathrm{CO}_{2}$ absorber and circulating pump had been removed. Details of the moving parts of the instrument, except the pulleys, are given in Table I.

\section{METHOD}

First the accuracy of the instrument as a volume recorder under static conditions was checked over the full range, from 0 to 6 litres, by introducing known small incremental volumes of air and recording the movements of the bell on the special charts provided.

Secondly the accuracy as a volume recorder under dynamic conditions was studied by measuring the excursion of the bell produced by pumping a constant volume of air into and out of the spirometer at different repetitive rates. In order to avoid alteration in rate or volume, or any subjective compensations for restraint offered by 
TABLE I

Physical Characteristics of Spirometers

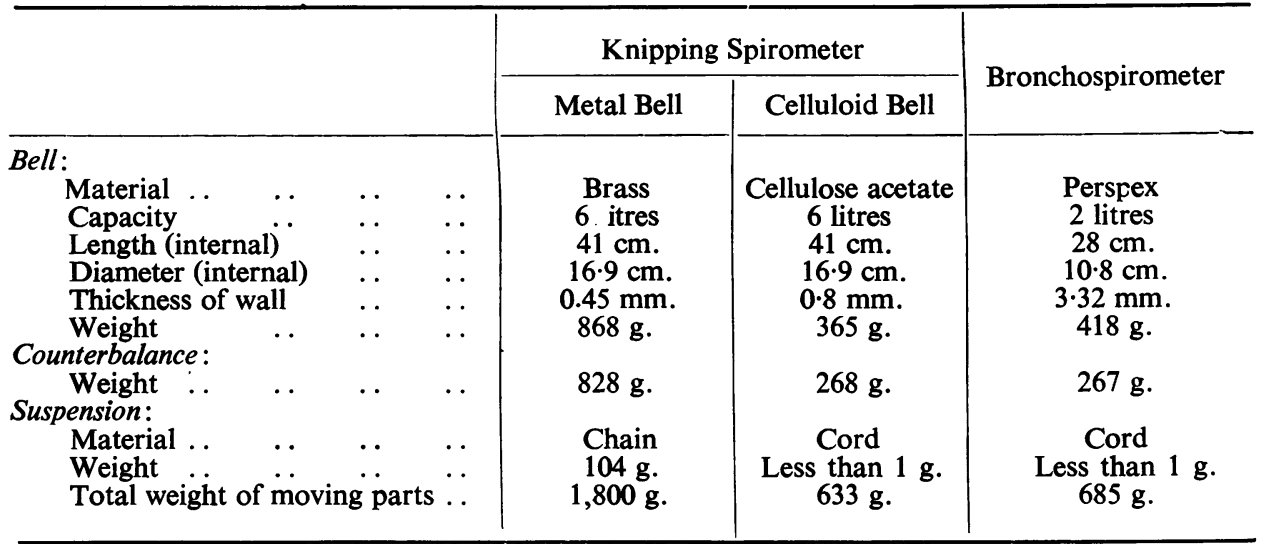

the instrument, a mechanical reciprocating pump was used instead of a human or animal subject. This pump (pump A) was of the piston type used as an artificial respiration pump in mammalian experiments, and the valves were made inoperative so that the same air was repeatedly injected into and withdrawn from the spirometer. The connexion between the pump and the spirometer was made by two corrugated rubber tubes, each $2 \mathrm{ft}$. long and 1 in. internal diameter. These connexions were identical with those used by D'Silva and Mendel for their M.B.C. determinations. Both tubes were used for both inflow and outflow. The stroke volume used was approximately $500 \mathrm{ml}$. This small volume was chosen because we wished to avoid any aspiration of water at high pump speeds which would have invalidated our measurements. (Experience in other fields suggests that provided a recording instrument is not overdriven its frequency response is independent of the amplitude of the oscillation under measurement.)

\section{RESUlts OF THE First EXPERIMENT}

Under Static Conditions.-The recorded excursion of the bell was found to be within $\pm 1.2 \%$ of the volume of air injected, over the full range of the instrument.

Under Dynamic Conditions.-The results of a single experiment are shown graphically in the heavy solid line graph of Fig. 1. The points at which observations were made have been joined by straight lines because the number of pump speeds available was too few to justify the drawing of a smooth curve. This graph is referred to hereafter as the frequency response curve of the spirometer. The errors in measurement were less than \pm 2 strokes per minute (s.p.m.) for pump frequency, and less than $\pm 10 \mathrm{ml}$. for the recorded excursion (equivalent to $\pm 2 \%$ of the pump output) and the results were closely repeatable.

It can be seen that at pump frequencies up to 45 s.p.m. the recorded excursion is constant and is equal to the known stroke volume of the pump. Above 45 s.p.m. it rises and at 58 s.p.m. it is $120 \%$ of the pump output. Above 58 s.p.m. it falls and at 71 s.p.m. it is only $40 \%$ of the pump output. Above 71 s.p.m. it rises again, until at 80 s.p.m. it has reached $70 \%$ of the pump output. It remains constant at this level until 102 s.p.m. is reached, and then falls again, to $50 \%$ of the pump output at 120 s.p.m. 


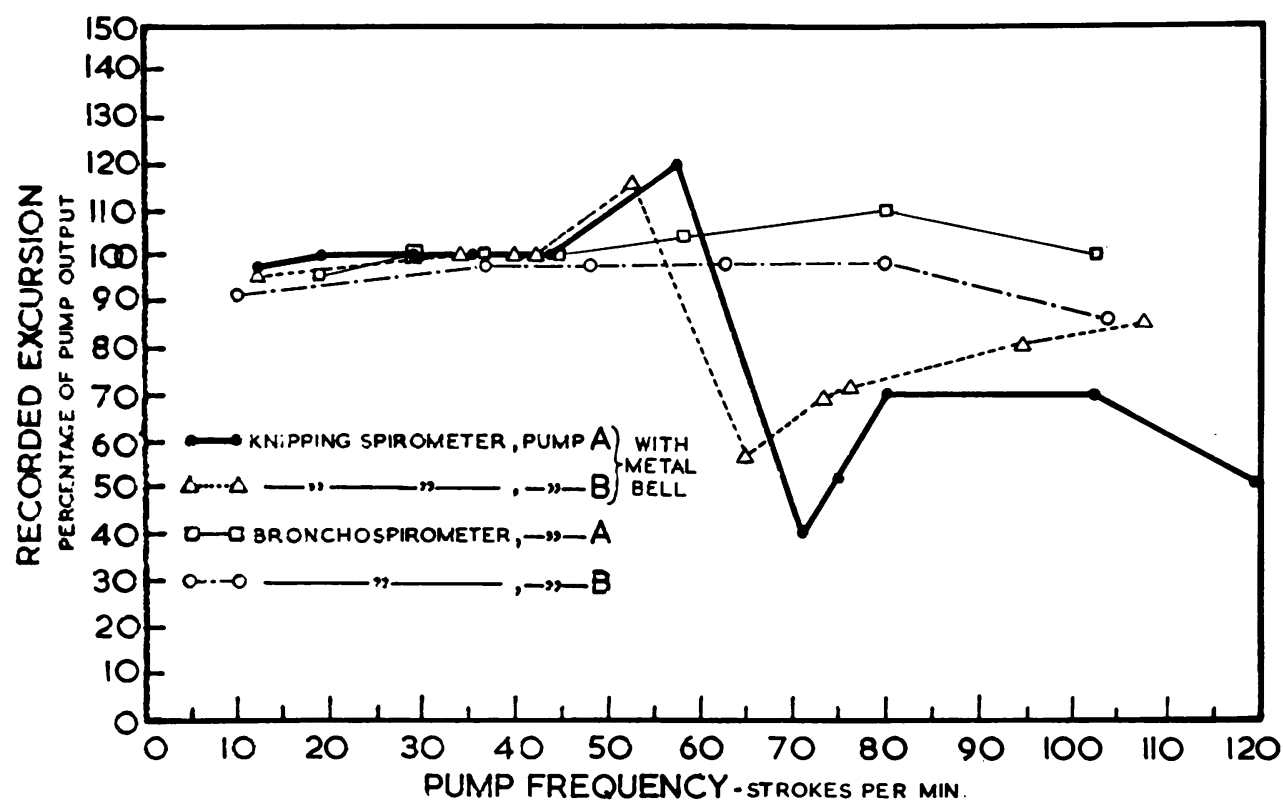

FIG. 1.-Frequency response curves of Knipping spirometer with metal bell, and of bronchospirometer, determined with pumps $\mathbf{A}$ and $\mathbf{B}$.

Although the spirometer gives an accurate recording of the volume of air introduced under static conditions, and also under dynamic conditions at frequencies below 45 s.p.m., it is apparent that there are considerable inaccuracies in the recorded excursions at the higher frequencies.

There are three possible causes for the discrepancies between the recorded excursions at high frequencies and the known stroke volume of the pump: (a) the spirometer may be unable to record accurately at these frequencies ; $(b)$ the pump may be unable to deliver its full stroke volume at these frequencies; and $(c)$ the rubber tubes connecting the spirometer and the pump may offer resistance to the air flow.

It would be easy to accept explanation $(b)$ or $(c)$ if the recorded excursion was always less than expected, but the high readings between 45 and 58 s.p.m. cannot be explained on either of these bases, and this suggests that explanation $(a)$ is the most likely to be correct. However, it was considered desirable to exclude the possibility that the pump or the connecting tubes were in any way responsible for the results, and therefore control experiments were performed using a second spirometer, and a second pump.

The second spirometer was a much smaller and lighter one which had been constructed for use in bronchospirometry. Details of the dimensions of the moving parts are given in Table I. This spirometer is referred to hereafter as "the bronchospirometer."

The second pump was also a reciprocating pump, but the dimensions of its cylinder were totally different from those of pump A, and it had a much greater reserve of driving power. This pump is referred to hereafter as " pump B." 


\section{METHOD FOR THE SECOND EXPERIMENT}

Frequency response curves were determined (1) for the Knipping spirometer, using pump B ; (2) for the bronchospirometer, using pump A; and (3) for the bronchospirometer, using pump B.

The connecting tubes used for the original experiment were used also for these experiments.

\section{RESUlTS OF THE SECOND EXPERIMENT}

These additional frequency response curves are also shown in Fig. 1.

Knipping Spirometer and Pump B.-This curve is very similar to that obtained with the same spirometer and pump A.

Bronchospirometer and Pump A.-This curve approximates much more closely to a straight line, and differs greatly from either of the curves for the Knipping spirometer.

Bronchospirometer and Pump B.-This curve is very similar to that obtained with the same spirometer and pump A. It differs greatly from either of the curves for the Knipping spirometer.

These results demonstrate that the shape of the frequency response curve is determined by the spirometer and not by the pump or by the connecting tubes used.

A possible explanation of the shape of the response curve of the Knipping spirometer is that the inertia of the moving parts is so great that they are unable to accommodate themselves sufficiently rapidly to the movements of the air at the higher frequencies, with the result that the excursion is reduced. This explanation would also account for the difference in behaviour from the bronchospirometer, whose moving parts are much lighter. It does not account for the increased excursion at frequencies near 58 s.p.m., and another factor must be invoked to explain this.

In order to determine the extent to which inertia of the moving parts was responsible for the shape of the response curve of the Knipping spirometer further experiments were performed using a much lighter bell and counterweight system in this spirometer.

\section{METHOD FOR THE THIRD EXPERIMENT}

A spirometer bell, of the same size and shape as the original metal one, was made from cellulose acetate sheet. A suitable counterweight was also made. With this bell and counterweight a suspension cord was used instead of a compensating chain. The resulting unbalance did not exceed $90 \mathrm{~g}$. in any position of the bell. This is equivalent to a pressure on the gas in the bell of just under $0.5 \mathrm{~mm}$. $\mathrm{Hg}$. We considered that for our purposes this slight disadvantage was negligible in view of the weight saved by not using a compensating chain. Details of the new bell and counterweight are given in Table $I$.

Using pump B, a frequency response curve for the Knipping spirometer with this new bell was determined. The records were made with the bell immersed in the water jacket of the spirometer to the same depth as the metal bell in the experiment with pump B.

\section{RESULTS OF THE THIRD EXPERIMENT}

The results of the experiment are shown in Fig. 2. The frequency response curve with the metal bell is superimposed for comparison. 


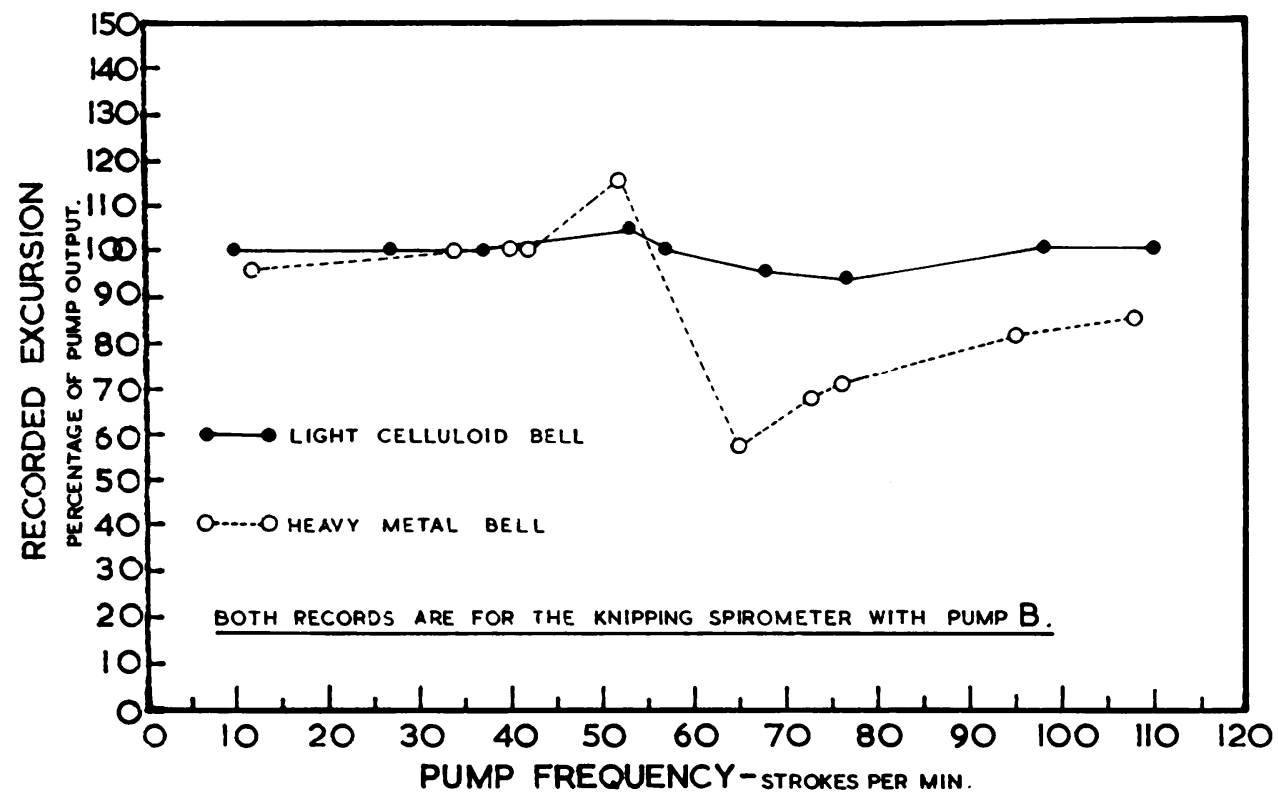

FIG. 2.-Frequency response curves of Knipping spirometer, using metal and celluloid bells, and determined with pump B.

The frequency response curve for the celluloid bell is considerably flatter than that for the metal bell, but it can be seen that the peak and trough in the curve do occur, and at the same frequencies, though they are much reduced in magnitude.

If the inertia of the moving parts were the sole cause of the irregularities in the frequency response curve, it would be anticipated that a reduction of their inertia should $(a)$ reduce the magnitude of the irregularities, and $(b)$ raise the frequency at which they occur.

In fact $(a)$ occurs but $(b)$ does not. It seems, therefore, that the inertia of the moving parts may excite some other component in the system which in turn determines the character of the irregularity, i.e., whether an increase or decrease in amplitude and the frequency at which it shall occur. Let us omit, for a moment, any consideration of this second factor. Then, at any frequency above that where inertia first limits the excursion of the bell, pressure changes must occur within the system because the change in volume at each excursion is less than that required to accommodate the output of the pump at atmospheric pressure. These pressure changes must cause movements of the water in the water jacket, which acts like a "U-tube" manometer in the system (Fig. 3). This water may then form an oscillating column.

To cause oscillation in this column of water two conditions must be satisfied: (a) each successive pressure change must cause slightly more movement of the water than can be damped out, between the pressure changes, by the inertia, viscosity and surface tension of the water, and $(b)$ the frequency of the exciting pressure changes must be at or near the natural resonant frequency of the column.

Provided these conditions are satisfied the column of water in the water jacket of the spirometer will tend to oscillate when the pump frequency is near the natural 


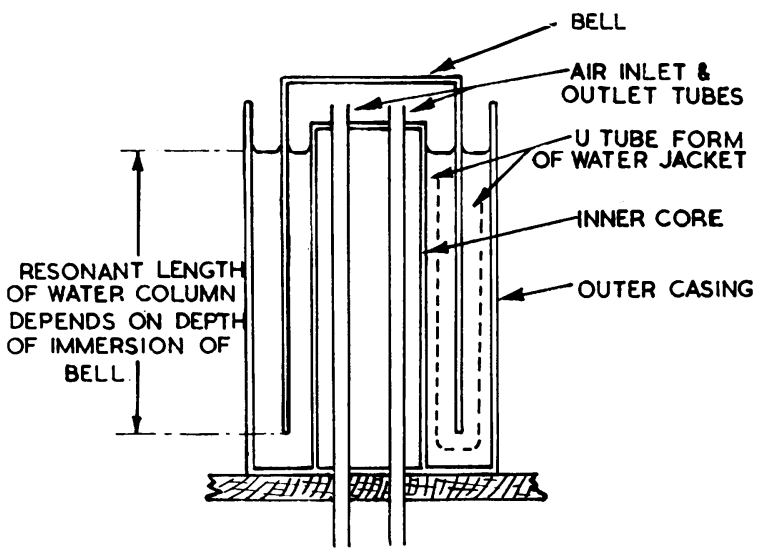

FIG. 3.-Sketch of spirometer to show how the water jacket effectively forms a U-tube in section, the length of the U-tube being determined by the depth of immersion of the bell.

resonant frequency of the column. The effect which these oscillations will have on the excursion of the spirometer bell will depend on the phase relations between the movements of each. It can be demonstrated mathematically that at the natural resonant frequency standing waves will occur in the water column but the excursions of the bell will be unaffected. Below the natural resonant frequency of the column the excursions of the bell will be increased, and above it they will be decreased. The oscillation of the column would then be the second factor, to which reference has already been made, which determines the character of the irregularities and the frequencies at which they occur, while the inertia of the moving parts of the spirometer would be responsible for the pressure changes which maintain the oscillation; it would thus determine the amplitude of the oscillation and hence the magnitude of the irregularities in the response curve.

If this hypothesis is correct two deductions can be made: (a) the use of a spirometer bell of identical dimensions but lighter in weight than that originally used in the Knipping spirometer should result in a frequency response curve in which the peak and trough occur at the same frequencies but are reduced in magnitude: it has been demonstrated by the previous experiment that this does occur; (b) an alteration in the length of the water column, by altering its natural resonant frequency, should alter the frequencies at which the peak and trough occur. An increase in length should lower the natural resonant frequency and so shift the irregularities toward the left (low frequency) end of the curve, and a decrease in length of the column should raise the natural resonant frequency and so shift the irregularities toward the right (high frequency) end of the curve. Experiments were conducted to test this deduction.

\section{METHOD FOR THE FOURTH EXPERIMENT}

Using pump B, frequency response curves were determined for the Knipping spirometer fitted with each bell in turn. For each bell three records were made: (1) with the bell almost totally immersed in the water jacket: this gave the longest possible water column ; (2) with the bell in the mid-position in the water jacket; and (3) with the bell only just immersed in the water jacket: this gave the shortest possible water column.

\section{Results OF THE Fourth EXPERIMENT}

The results of this experiment are shown in Fig. 4. 


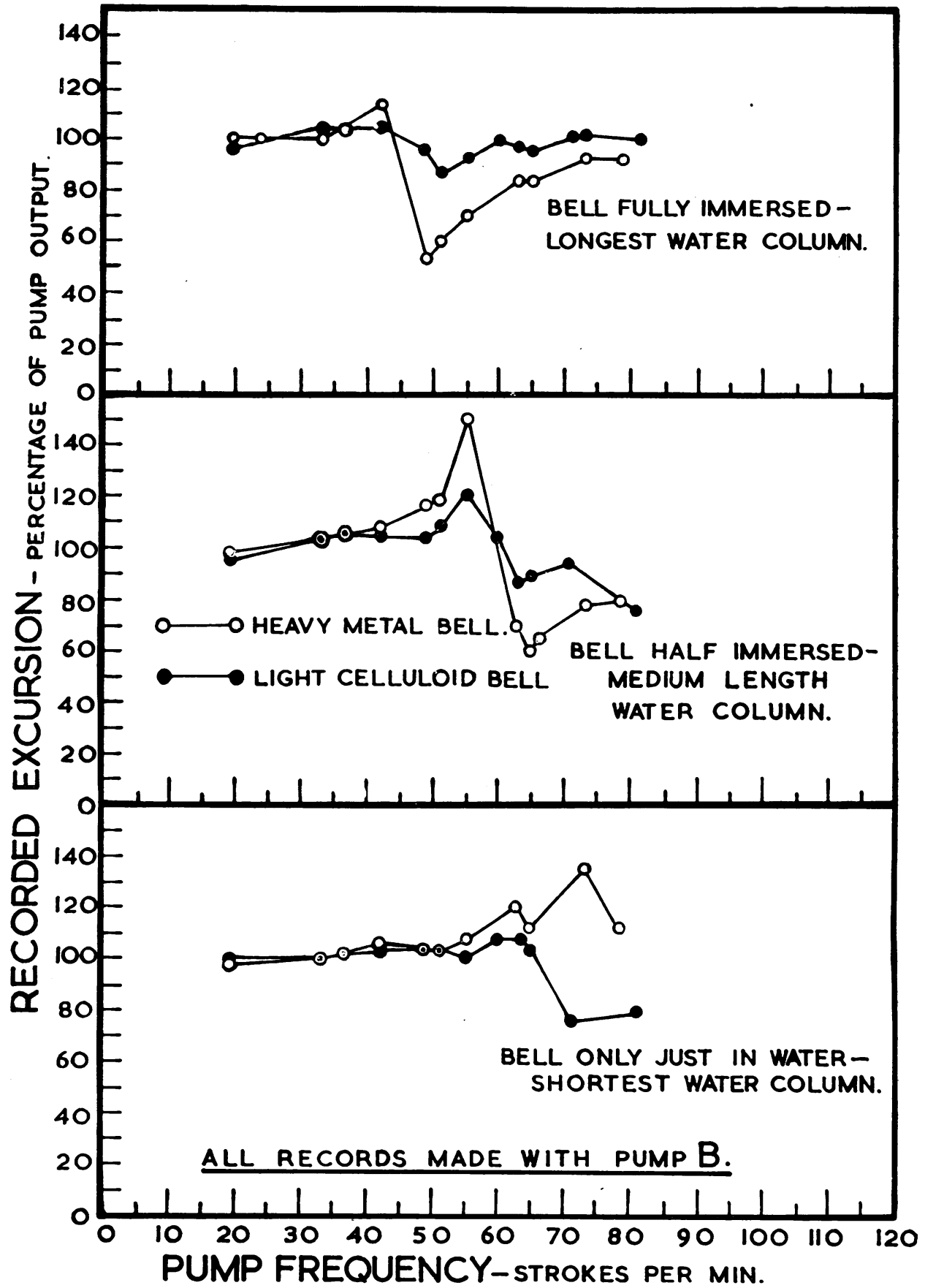

Fig. 4.-Frequency response curves of Knipping spirometer, using the metal and the celluloid bells, and with the bell immersed in the water jacket to three different depths. 
For any given length of water column the curves for the two bells show a general similarity in shape. The peak and trough occur at the same frequencies for each bell, but are reduced in magnitude with the celluloid bell.

Comparing the records made with the longest and the medium length water columns, it is evident that shortening the column causes the critical frequencies to shift toward the right (high frequency) end of the curve. The record made with the shortest water column does not show any definite peak or trough, so that an exact determination of the critical frequencies is not possible in this case. However, the absence of these phenomena in the frequency range where they occur with the longer water columns itself suggests that a further shift to the right (high frequency) end has occurred and that our records do not extend far enough in this direction. Unfortunately, mechanical difficulties with the pump precluded any records being made at higher frequencies.

It will be seen that the results of this experiment confirm those of the previous experiment, namely, that the reduction of the inertia of the moving parts of the spirometer reduces the magnitude of the irregularities in the response curve but does not alter their character or the frequencies at which they occur. In addition they confirm the deduction that the frequencies and character of the irregularities in the response curve are determined by the length of the resonating water column in the water jacket. They are therefore in agreement with the hypothesis which has been advanced to explain these irregularities.

In every experiment performed it was observed that the oscillation in the water column was at its greatest amplitude when the pump frequency was in the range where the response curve of the spirometer is non-linear. In each case the oscillation was smaller with the light celluloid bell than with the heavier metal one. These observations are in accordance with the hypothesis.

If the pump is set in motion at a frequency which is known to produce an inaccurate spirometer excursion, the hypothesis demands that the excursions should change, from an initial accurate amplitude to the final inaccurate one, as the oscillation in the water column builds up. Fig. 5 shows two records-one of the generation of an increased excursion, the other of the generation of a diminished excursion-and illustrates that this does occur as predicted.

It may be objected that these results are not applicable to records of human tidal air for the following reasons:

The graph, with respect to time, of the output of a reciprocating pump is a sine wave. 'The maximum effort tidal air tracing of a human subject is not sinusoidal and may be "a triangular pattern when the emphasis is placed on rapidity, and a nearly rectangular one when the emphasis is on depth" (Proctor and Hardy, 1949). Therefore the phenomena in maximum tidal air recordings are not of the same kind as with the pump.

The experiments were all made with a pump output of only $500 \mathrm{ml}$. Tidal air records are usually of much greater amplitude. Therefore conclusions from the experiments with small volumes may not be applicable.

With regard to the first objection, it can be shown, by Fourier analysis, that any non-sinusoidal waveform can be considered as being the algebraic sum of two or more component sine-waves. The component of lowest frequency (i.e., the fundamental) determines the repetition rate of the complex waveform. The other 


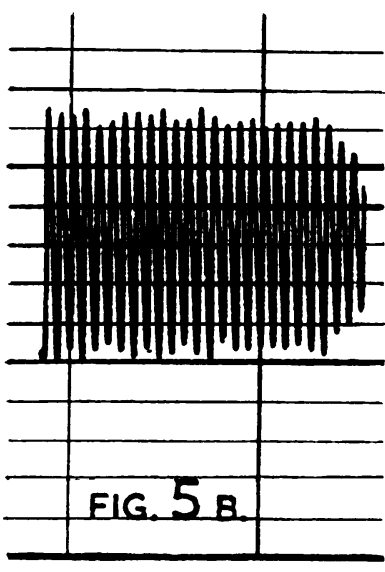

75 S.P.M.

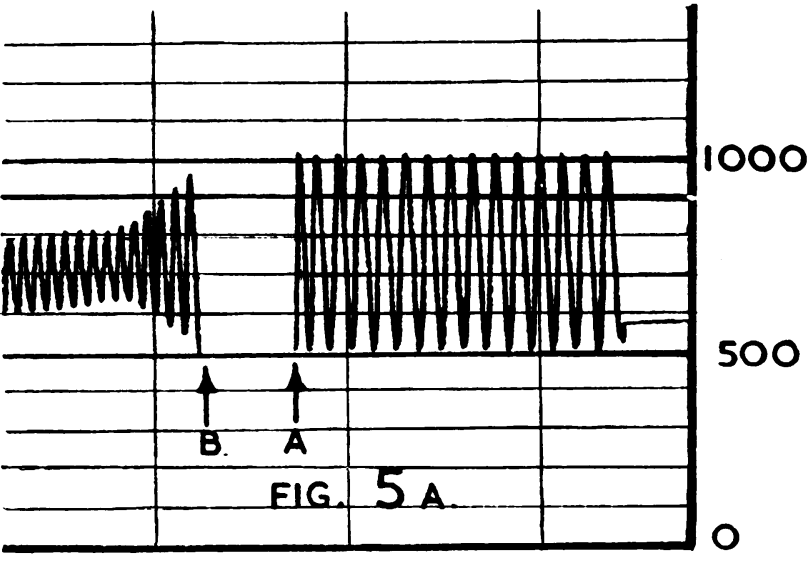

7I S.P.M.
45 S.P.M.

\section{RECORD READS FROM RIGHT TO LEFT. VERTICAL LINES REPRESENT 0.2 MIN. INTERVALS.}

FIG. 5a.-From the start of the recording to the point $A$ the record shows the accurate excursion of - the bell with a pump output of $500 \mathrm{ml}$. at a frequency of 45 s.p.m. At $A$ the pump was stopped, and it was restarted at B, at 71 s.p.m., but with the same stroke volume. The first excursion after the restart is nearly accurate, but as the water column is excited into oscillation the excursion is reduced.

FIG. 5b.-This shows how an increased inaccurate excursion was built up from an initially accurate one. The pump output was $500 \mathrm{ml}$., the rate was 75 s.p.m., but the bell was less deeply immersed than in the experiment of Fig. $5 a$.

components have frequencies which are multiples of the fundamental. Any deviation of the tidal air curve from a true sine-wave must be due to the existence of components at frequencies which are multiples of the respiratory rate. The response of the spirometer to these higher frequency components may be expected to be even less accurate than to the fundamental frequency. In so far as respiratory airflow differs from the sinusoidal output of the reciprocating pump, the records made with the spirometer can be expected to show greater inaccuracies at high respiratory rates.

\section{METHOD FOR FURTHER EXPERIMENTS}

To determine the validity, or otherwise, of the second objection further experiments were made with a pump capable of delivering an output of $2,830 \mathrm{ml}$. (pump C). This was a large piston-type pump known to be leak-proof. The pump was manually operated and the various frequencies were obtained by pumping to the rhythm of a metronome, which was adjusted appropriately. The output was maintained constant by always pumping the full stroke volume. Frequency response curves were determined for the Knipping spirometer using the metal and the light celluloid bells in turn.

\section{RESULTS OF FURTHER EXPERIMENTS}

The results are shown in the graphs of Fig. 6 . 


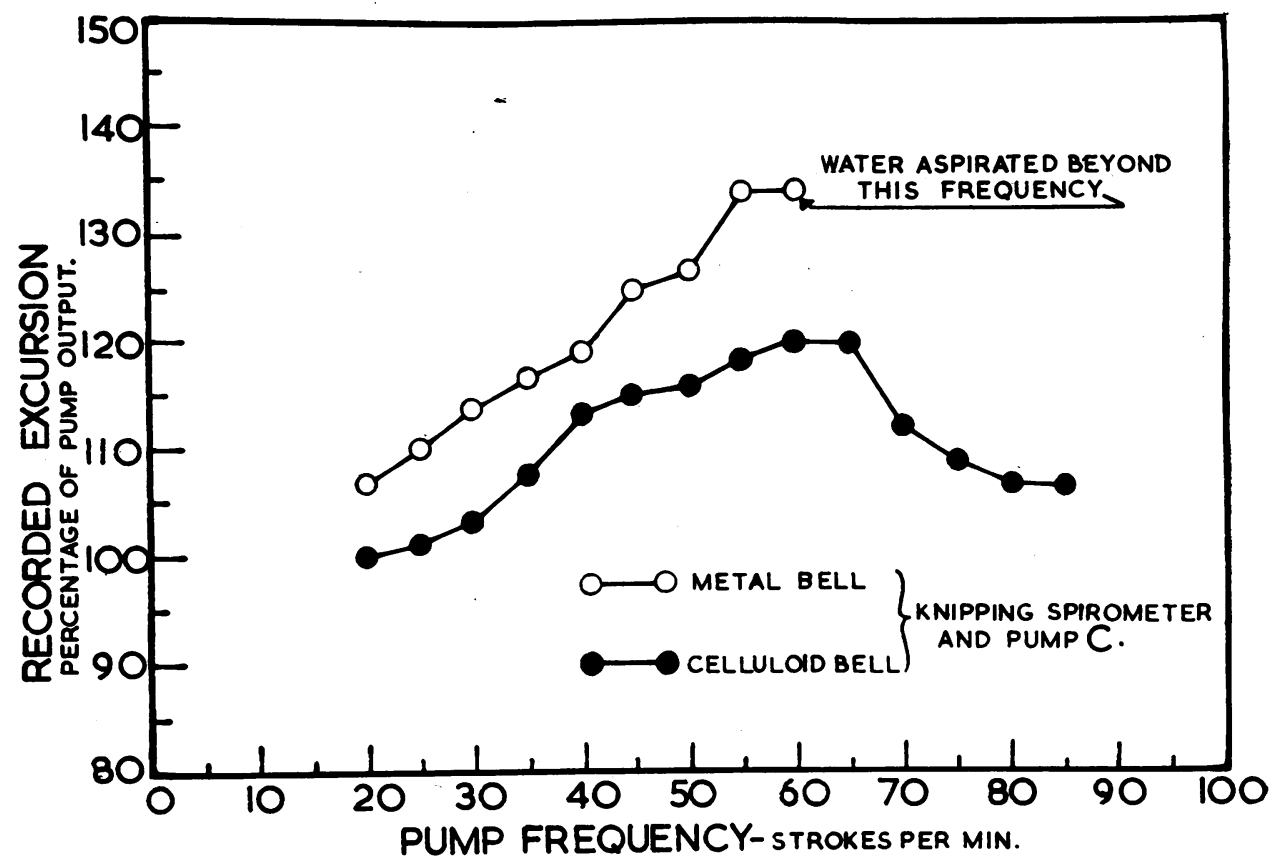

FIG. 6.-Frequency response curves of Knipping spirometer, using the metal and the celluloid bells, determined with pump $C$ (pump output $2,830 \mathrm{ml}$.). The waveform of the airflow is not sinusoidal, and these curves are therefore not comparable directly with those of Figs. 1, 2, and 4.

With the Metal Bell.-There is a steady increase in recorded amplitude as the frequency rises. At 65 s.p.m. water was aspirated into the connecting tubes, and for this reason no records were possible at this or any higher frequency.

With the Celluloid Bell.-There is an increase in recorded amplitude from 20 s.p.m. to 60 s.p.m. after which the recorded amplitude falls again until the highest frequency, 85 s.p.m., is reached. Records were not possible at any higher frequency because of the difficulty in working the pump at such speeds. Aspiration of water did not occur at any frequency in the recorded range.

These results demonstrate that the non-linearity of the frequency response curve of the spirometer applies to large as well as to small excursions; the results with the light celluloid bell are better than with the metal one in two respects (1) no water is aspirated and (2) the errors are reduced.

Fig. $7 a$ is a typical record made with the metal bell for a single maximal "expiration" followed by a single maximal "inspiration" of the pump. It can be seen that the bell overshoots the final recorded position at the end of each movement and executes an exponentially damped oscillation before it comes to rest. These oscillations of the bell were observed to be in exact correspondence with movements of the water in the water jacket. With the celluloid bell similar oscillations occur, but the amplitude is much reduced (Fig. $7 b$ ). The frequencies of these free oscillations were measured. The results are given in Table II. 
TABLE II

Frequency of Natural Oscillations of Spirometer Bell

\begin{tabular}{|c|c|c|c|c|c|}
\hline \multirow{2}{*}{\multicolumn{3}{|c|}{$\begin{array}{c}\text { Position of } \\
\text { Spirometer Bell }\end{array}$}} & \multirow{3}{*}{$\begin{array}{c}\begin{array}{c}\text { Length of } \\
\text { Water Column }\end{array} \\
\text { Short }\end{array}$} & \multicolumn{2}{|c|}{ Frequency (cycles per minute) } \\
\hline & & & & Metal Bell & Celluloid Bell \\
\hline Top of Excursion & 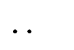 & . & & $72 \pm 3 \cdot 5$ & $80 \pm 4$ \\
\hline Bottom of Excursion & - & . & Long & $55 \pm 2 \cdot 5$ & $60 \pm 3$ \\
\hline
\end{tabular}

The figures in columns 3 and 4 include the estimated errors in measurement of the frequency.

The frequency was determined by measuring the length occupied on the tracing by not fewer than 3 oscillations. Error was introduced into this measurement chiefly by the thickness of the recorded line. The estimate of the accuracy of the determination of the frequency was based upon an estimate of this error in linear measurement.

It was not possible to achieve great accuracy in these measurements because only three or four cycles were recorded before the oscillations died out, but, within the limits of accuracy obtained, the frequency is the same for both bells, given the same length of water columns, and increases as the water column is shortened.

These observations and measurements afford direct confirmation of the hypothesis concerning the causes of the irregularities in the response curve of the spirometer. They demonstrate that the frequency of the free oscillations of the bell, at the end of a single "expiration" or "inspiration," is determined only by the length of the water column, and that their amplitude is determined by the inertia of the moving parts of the spirometer.

Fig. $7 c$ is a record of the excursion at a pump frequency of 20 s.p.m. (pump C). During the making of the record two methods of pumping were used. (1) An attempt was made to obtain a sinusoidal airflow similar to that given by pumps A and B, and (2) the individual "inspirations" and "expirations" were executed as rapidly as possible with a pause between each, in an attempt to produce a non-sinusoidal airflow.

It can be seen that the excursion with the approximately sinuscidal airflow is accurate and that there is little tendency to oscillation at the end of "inspiration" and "expiration." With the non-sinusoidal airflow oscillations occur at the end of each stroke, and cause an inaccurate recorded excursion.

These results confirm the hypothesis that records of human respiration-in which the air flow is non-sinusoidal-may be even less accurate than the results of the experiments with pumps $A$ and $B$ would suggest.

The results of these additional experiments justify the conclusion that the inaccuracies in spirographic recording, as revealed by the experiments with reciprocating pumps, occur also in measurements of larger tidal air volumes.

\section{Discussion}

Baldwin, Cournand, and Richards (1948) have foreseen the possibility that the inertia of the spirometer bell, and the frictional resistance of the recording devices, may cause some reduction in the recorded excursion at high respiratory rates, but they state that comparative studies with the spirographic and another method of measurement have failed to reveal any significant discrepancy. 
FIG.7A. FIG 7B

FIG 7C

BELL AT BOTTOM OF EXCURSION

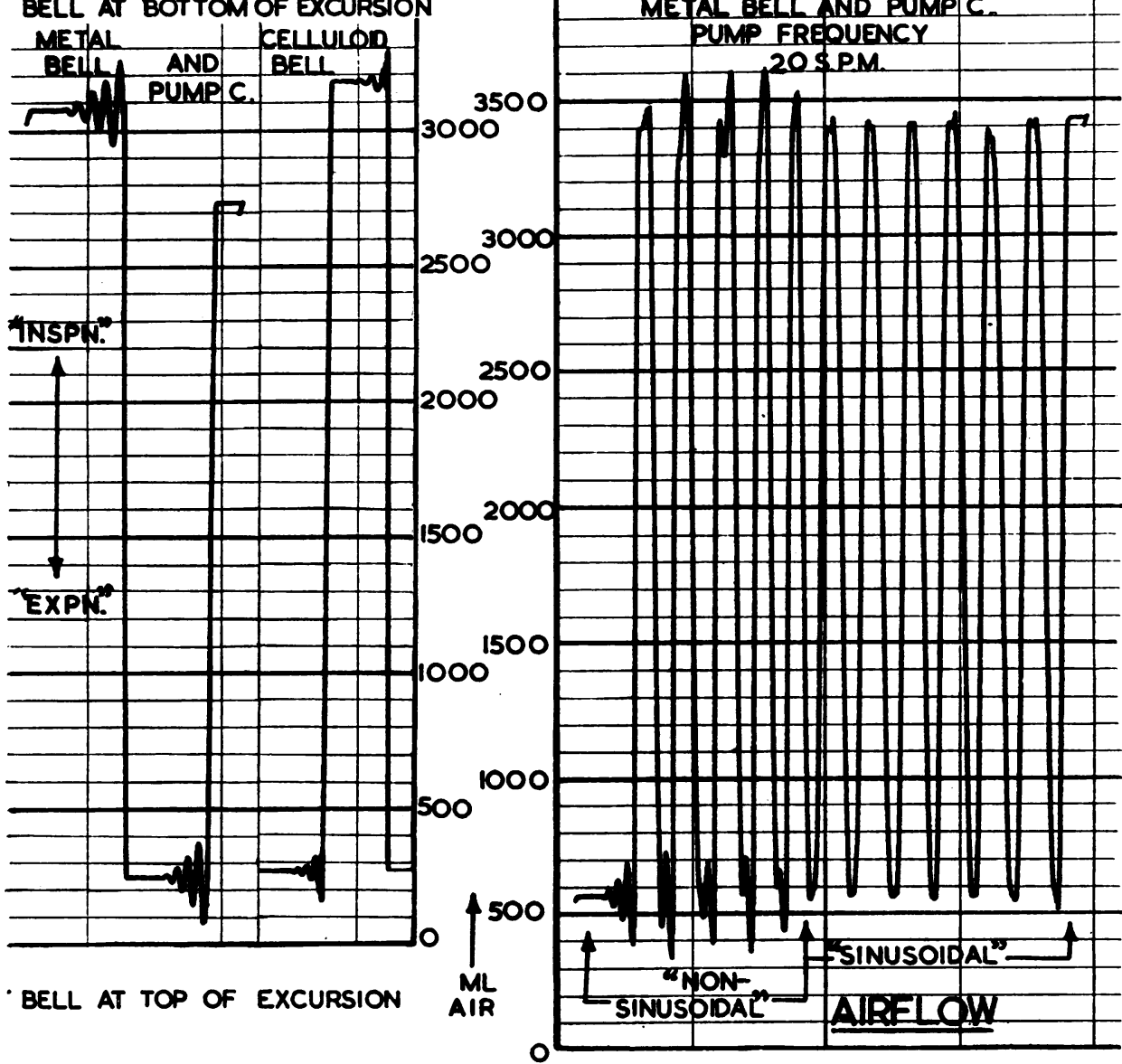

RECORDS READ FROM RIGHT TO LEFT.

VERTICAL LINES REPRESENT 0.2 MIN. INTERVALS.

Fig. 7a.-This shows the oscillations of the metal bell at the end of a single "expiration" and at the end of a single "inspiration" of pump C.

Fig. 7b.-A similar record made with the celluloid bell.

FIG. 7c.-This shows how the record is accurate with an approximately sinusoidal airflow at 20 s.p.m. When the airflow is changed to a non-sinusoidal form, oscillations occur at each phase reversal, and these cause the recording to be grossly inaccurate.

In our experiments it has been demonstrated that even though a spirometer may be accurate under static conditions, it may give grossly inaccurate records at higher rates of respiration. The errors measured are not directly applicable to all spirometers, but the defects in design which cause them are inherent in most spirometers. 
It has been shown that at least two factors are concerned in the non-linearity of the frequency response curve of a spirometer: $(a)$ the inertia of its moving parts, and $(b)$ the phenomenon of resonance in the water column in the water jacket. It may be that other factors as well as these are involved, but our experiments do not demonstrate them.

For the following reasons, it is not practicable to draw a calibration curve for a spirometer so that the recording errors may be corrected.

With a small tidal air the errors depend on the mean position of the bell in the water jacket. A complete family of calibration curves will be necessary unless all records are made with the bell moving about the same mean position.

The errors depend to a considerable extent on the waveform of the respiratory airflow. Without a detailed analysis of the waveform in every recording it is not possible to determine the correction factors required.

The greatly improved response curves obtained with the lightweight celluloid bell suggest that the moving parts should be kept as light as possible in spirometers used at high rates of breathing.

\section{SUMMARY}

A spirometer may not give a record of constant amplitude for a constant tidal air at all respiratory rates.

Two factors, at least, are responsible for this: $(a)$ inertia of the moving parts of the spirometer, and $(b)$ resonance of the water column in the water jacket.

The frequency response depends on the waveform of the airflow being measured. Because respiratory airflow may vary in waveform, there is no simple way of calibrating the spirometer.

Considerable improvement results if the moving parts are made as light as possible.

We wish to thank Prof. J. L. D'Silva for suggesting this investigation, and for his help and criticism; Prof. R. V. Christie for his criticism and advice, and for the loan of the large capacity pump; and Mr. J. Young for the construction and adjustment of apparatus.

\section{REFERENCES}

Baldwin, E. de F., Cournand, A., and Richards, D. W. (1948). Medicine, Baltimore, $27,243$.

D'Silva, J. L., and Mendel, D. (1950). Thorax, 5, 325.

Hermannsen, J. (1933). Z. ges. exp. med., 90, 130.

Proctor, D. F., and Hardy, J. B. (1949). Bull. Johns Hopk. Hosp., $85,253$. 